\title{
On the Zeros of the Riemann Zeta Function in the Critical Strip. II
}

\author{
By R. P. Brent, J. van de Lune, H. J. J. te Riele and D. T. Winter
}

\begin{abstract}
We describe extensive computations which show that Riemann's zeta function $\zeta(s)$ has exactly 200,000,001 zeros of the form $\sigma+i t$ in the region $0<t<81,702,130.19$; all these zeros are simple and lie on the line $\sigma=\frac{1}{2}$. (This extends a similar result for the first $81,000,001$ zeros, established by Brent in Math. Comp., v. 33, 1979, pp. 1361-1372.) Counts of the numbers of Gram blocks of various types and the failures of "Rosser's rule" are given.
\end{abstract}

1. Introduction. Riemann's zeta function is the meromorphic function $\zeta: \mathbf{C} \backslash\{1\} \rightarrow$ C, which, for $\operatorname{Re}(s)>1$, may be represented explicitly by

$$
\zeta(s)=\sum_{n=1}^{\infty} n^{-s} \quad(s=\sigma+i t) .
$$

It is well known (see Titchmarsh [16, Chapters II and X]) that

$$
\xi(s):=\frac{1}{2} s(s-1) \pi^{-s / 2} \Gamma(s / 2) \zeta(s)
$$

is an entire function of order 1 , satisfying the functional equation $\xi(s)=\xi(1-s)$, so that

$$
\Xi(z):=\xi\left(\frac{1}{2}+i z\right) \quad(z \in \mathbf{C}),
$$

being an even entire function of order 1 , has an infinity of zeros. The Riemann Hypothesis is the statement that all zeros of $\Xi(z)$ are real, or, equivalently, that all nonreal zeros of $\zeta(s)$ lie on the "critical" line $\sigma=\frac{1}{2}$. Since $\zeta(\bar{s})=\overline{\zeta(s)}$, we may restrict ourselves to the half plane $t>0$. To this day, Riemann's Hypothesis has neither been proved nor disproved.

Numerical investigations related to this unsolved problem were initiated by Riemann himself and later on continued more systematically by the writers listed below (including their progress).

\begin{tabular}{llc}
\hline Investigator & Year & $\begin{array}{c}\text { The first } n \text { complex zeros of } \zeta(s) \\
\text { are simple and lie on } \sigma=\frac{1}{2}\end{array}$ \\
\hline Gram [6] & 1903 & $n=15$ \\
Backlund [1] & 1914 & $n=79$ \\
Hutchinson [7] & 1925 & $n=138$ \\
Titchmarsh [15] & $1935 / 6$ & $n=1,041$ \\
\hline
\end{tabular}

Received January 15, 1982.

1980 Mathematics Subject Classification. Primary 10H05; Secondary 10-04, 65E05, 30-04.

Key words and phrases. Gram blocks, Riemann hypothesis, Riemann zeta function, Riemann-Siegel formula, Rosser's rule. 
Those listed above utilized the Euler-Maclaurin summation formula and performed their computations by hand or desk calculator, whereas those listed below applied the Riemann-Siegel formula in conjunction with electronic computing devices.

\begin{tabular}{lll}
\hline Lehmer [10], [11] & 1956 & $n=25,000$ \\
Meller [13] & 1958 & $n=35,337$ \\
Lehman [9] & 1966 & $n=250,000$ \\
Rosser, Yohe \& Schoenfeld [14] & 1968 & $n=3,500,000$ \\
Brent [2] & 1979 & $n=81,000,001$ \\
\hline
\end{tabular}

An excellent explanatory account of most of these computations may be found in Edwards [4].

In this paper, which should be considered as a continuation of Brent [2]*, we report on extensive computations by which the first named author has extended his former result to $n=156,800,001$ and by which the remaining three authors (L R \& $\mathrm{W}$, for short) have extended this bound to $n=200,000,001$. Details of the last result, together with a full program listing, are given in van de Lune, te Riele and Winter [12]. Independently of Brent, L R \& W have also checked the range $\left[g_{81,000,000}\right.$, $\left.g_{120,000,000}\right)$.

In practice, the numerical verification of the Riemann hypothesis in a given range consists of separating the zeros of the well-known real function $Z(t)$ (see formula (2.6) of Brent [2]), or, equivalently, of finding sufficiently many sign changes of $Z(t)$. Our programs (aiming at a fast separation of these zeros) are based, essentially, on the modification of Lehmer's [11] method introduced by Rosser et al. [14]. L R \& W have developed a more efficient strategy of searching for sign changes of $Z(t)$ in Gram blocks of length $L \geqslant 2$. Brent's average number of $Z$-evaluations, needed to separate a zero from its predecessor, amounts to about 1.41 (compare Brent [2]), whereas L R \& W have brought this figure down to about 1.21. It may be noted here that in the most recent version of the program of $L R \& W$ this figure has been reduced further to about 1.185. From the statistics in Section 4, it follows that in the range $\left[g_{156,800,000}, g_{200,000,000}\right)$ this average number of $Z$-evaluations could not have been reduced below 1.135 by any program which evaluated $Z(t)$ at all Gram points. We also note that about 98 percent of the running time of the L R \& W-program was spent on evaluating $Z(t)$. This program was executed on a CDC CYBER 175 computer and ran about ten times as fast as the UNIVAC $1100 / 42$ program of Brent. This is roughly what could be expected, given the relative speeds of the different machines.

2. The Strategy for Finding the Required Number of Sign Changes of $Z(t)$ in a Gram Block of Length $L \geqslant 2$. The strategy of Brent for finding the required number of sign changes of $Z(t)$ is exactly as described in Section 4 of Brent [2]. L R \& W refined this strategy in order to reduce the number of $Z$-evaluations as much as they could. This will be described here in some detail.

\footnotetext{
* We take the opportunity to make the following corrections in Brent [2]: on p. 1361, line $9 \uparrow$, replace " $H(10)$ " by " $H(15)$ "; on p. 1362 , Eq. (2.3), replace " $B_{2 k}$ " by " $\left|B_{2 k}\right|$ ".
} 
In order to reduce the number of $Z$-evaluations as much as possible, we first observe that after having determined a Gram block $B_{j}$ of length $L \geqslant 2$, we already have implicitly detected $L-2$ sign changes of $Z(t)$. Hence, the problem reduces to finding the "missing two" sign changes. Next we observe that these missing two (if existing) must both lie in one and the same Gram interval of the block $B_{j}$. Some preliminary experiments with the $\mathrm{L} R \& \mathrm{~W}$-program revealed that in the majority of cases the missing two are situated in one of the outer Gram intervals of $B_{j}$. Therefore, we first search in $\left(g_{j}, g_{j+1}\right)$ or $\left(g_{j+L-1}, g_{j+L}\right)$ according to which of $\operatorname{abs}\left(Z\left(g_{j}\right)+Z\left(g_{j+1}\right)\right)$ and $\operatorname{abs}\left(Z\left(g_{j+L-1}\right)+Z\left(g_{j+L}\right)\right)$ is the smallest. In the selected interval an efficient parabolic interpolation search routine is invoked. (Here is the main improvement over Brent's method, which used random search rather than parabolic interpolation.) If this routine terminates without having found the missing two sign changes, the other outer Gram interval of the block is treated in the same manner. In case the missing two are still not found, another search routine is called, depending on the length $L$ of the block $B_{j}=\left[g_{j}, g_{j+L}\right)$.

If $L=2$, the interval $\left(g_{j}, g_{j+2}\right)$ is scanned again, and if $L>2$, we continue to search in the interval $\left(g_{j+1}, g_{j+L-1}\right)$. In both cases, the search is performed by means of a refinement of a search routine described by Lehman [9]. For more details we refer the reader to the source text of the L R \& W-program in [12].

If at some instant one of the search routines has detected the missing two, a new Gram block is set up, and we continue as described above. In the opposite case the program prints a message and a "plot" of $Z(t)$ corresponding to the whole Gram block under investigation and proceeds by pretending (!) that the missing two were found indeed. These plots of $Z(t)$ were inspected afterwards (if necessary) "by hand". So far, the missing two were always easily found either in the Gram block under consideration or in an adjacent Gram block; compare Brent [2, Section 4].

After having covered the range $\left[g_{156,800,000}, g_{200,000,000}\right)$, we ran the computation a little further and found 4 Gram blocks in $\left[g_{200,000,000}, g_{200,000,004}\right)$, all of them satisfying Rosser's rule. By applying Theorem 3.2 of Brent [2], we completed the proof of our claim that the first $n=200,000,001$ zeros of $\zeta(s)$ are simple and lie on $\boldsymbol{\sigma}=\frac{1}{2}$.

We (L R \& W) intend to extend our computations in the near future.

3. Computation of $Z(t)$ and Error Analysis. In principle, Brent's and L R \& W's methods of computing $Z(t)$ and error analysis are exactly as described in Section 5 of Brent [2]. We shall only mention here the differences between $L$ R \& W's computations and error analysis and Brent's. Details are given in [12].

The L R \& W-computations were carried out on a CDC CYBER 175 computer having a 60-bit word, and single-precision and double-precision floating point arithmetic using 48- and 96-bit binary fractions, respectively. The function $Z(t)$ was computed (in both methods A and B) using the Riemann-Siegel formula with two terms in its asymptotic expansion. Gabcke's error bound (see [5]) $\left|R_{1}(t)\right|<$ $0.053 t^{-5 / 4}$, for $t \geqslant 200$, was used. The same bound is also given as a special case of more general bounds in Brent and Schoenfeld [3]. For method A, a precomputed table of 8194 cosine-values, and a precomputed table of 8193 corresponding differences of cosine-values, was used in the linear interpolation formula for the cosine-approximation. The main loop in method A was programmed in machine 
language (COMPASS), and one cycle of this loop executed in about $2.1 \mu \mathrm{sec}$. The error analysis, accounting for all possible errors in the machine computation of $Z(t)$, was carried out for any $t$ in the interval $\left(3.5 \times 10^{7}, 3.72 \times 10^{8}\right)$. This interval covers the range of zero \#81,000,000 till zero \#1,000,000,000 of $\zeta(s)$ in the critical strip, which $\mathrm{L}$ R \& W originally planned to investigate. The following bounds for the error in the computed value $\tilde{Z}(t)$ of $Z(t)$ were derived:

$$
|\tilde{Z}(t)-Z(t)| \leqslant\left\{\begin{array}{r}
3 \times 10^{-7} \tau^{1 / 4} \text { for method } A,{ }^{* *} \\
\left(5.4 \times 10^{-3} \tau^{-3 / 2}+3.1 \times 10^{-16}+4.1 \times 10^{-24} \tau^{-1 / 2}\right. \\
\left.+5 \times 10^{-26} \tau \ln (\tau)\right) \tau^{1 / 4} \text { for method B }
\end{array}\right.
$$

for any $t(=2 \pi \tau)$ in the interval $\left(3.5 \times 10^{7}, 3.72 \times 10^{8}\right)$. In this interval, a safe upper bound for the error is $2.7 \times 10^{-5}$, respectively $2.0 \times 10^{-11}$. In the $\mathrm{L} \mathrm{R} \&$ $\mathrm{W}$-program, the extremely conservative fixed bounds $10^{-4}$ (and sometimes even $2 \times 10^{-4}$ ) respectively $2.5 \times 10^{-6}$ were used. Nevertheless, until now not a single $t$ was met for which method B could not determine the sign of $Z(t)$ rigorously.

4. Statistics. The L R \& W-program was organized in such a way that in case the value of $Z(t)$, obtained with method $\mathrm{A}$, was too small for a rigorous sign determination, a few small shifts of the argument were tried before method B was invoked. Therefore, the L R \& W-program uses, in relatively few cases, an approximation to the Gram point $g_{j}$ instead of $g_{j}$ itself. (In a run of 2,500,000 zeros, with error bound $10^{-4}$ for method A, the total number of shifts was always less than 370 . Most of them were made when separating the zeros inside the Gram blocks. Only a few of them were made in Gram points. Also see the text introducing Table 3.) Consequently, the statistics found by L R \& W cannot, strictly speaking, be accumulated to those found by Brent. Nevertheless, just for convenience, we have put together all results. This should be kept in mind when reading the tables.

In Table 1 we present a list of 104 exceptions to Rosser's rule up to $g_{200,000,000}$ found by Brent and L R \& W, including the 15 exceptions up to $g_{75,000,000}$ from [2], for completeness. Moreover, the types are given in parentheses, followed by the local extreme values of $S(t)$ near $B_{n}$. It is possible that for $n \geqslant 156,800,000$ the $\mathrm{L} \mathrm{R} \&$ $\mathrm{W}$-program has not detected all exceptions to Rosser's rule, due to possible shifts in Gram points. For instance, an exception of type 2 (see Table 2) may have been detected as a Gram block of length 3 with " 210 " zero-pattern. It may be noted, however, that in the range $\left[g_{81,000,000}, g_{120,000,000}\right)$ L R \& W have found exactly the same exceptions to Rosser's rule as Brent.

In addition to the types 1, 2, and 3 introduced by Brent [2], we have defined the types 4,5 , and 6 , the meaning of which should be clear from Table 2 . This table also gives the frequencies of the occurrences of the various types in $\left[g_{-1}, g_{200,000,000}\right)$. Note that an exception of type 4 has not yet been found, so that at the time of writing we still know only one Gram interval with four zeros, viz. $G_{61,331,768}$, found by Brent [2].

\footnotetext{
** In [12] a four-term bound was given for method A. A closer look at the error analysis led us to the simpler bound given here.
} 


\section{TABLE 1}

(extension of Table 3 of Brent [2])

\section{4 exceptions to Rosser's rule up to $g_{200,000,000}$}

Notation: $n$ (type) extreme $S(t)$ where $n$ is the index of the Gram block $B_{n}$ containing no zeros.

\begin{tabular}{|c|c|c|c|c|c|c|c|}
\hline $5(1)$ & -2.0041 & $100,788,444(1)$ & -2.0230 & $146,130,246(2)$ & 2.0005 & $173,737,614(2)$ & 1 \\
\hline $3,329(1)$ & -2.0026 & $106,236,172(1)$ & -2.0184 & $147,059,770(1)$ & -2.0498 & $174,102,513(1)$ & -2.0180 \\
\hline $30,930,927(2)$ & 2.0506 & $106,941,328(2)$ & 2.1559 & $147,896,100(2)$ & 2.0391 & $174,284,990(1)$ & -2.0181 \\
\hline $37,592,215(1)$ & -2.0764 & $107,287,955(1)$ & -2.0786 & $151,097,113(1)$ & -2.0043 & $174,500,513(1)$ & -2.0125 \\
\hline $40,870,156(1)$ & -2.0038 & $107,532,017(2)$ & 2.0728 & 1 & -2.0026 & $09(1)$ & -2.0193 \\
\hline 43 & -2.0242 & $110,571,044(1)$ & -2.0458 & $3,169(2)$ & 2.0459 & $176,870,844(2)$ & 2.0125 \\
\hline 46, & 311 & $111,885,254(2)$ & 2.0247 & $153,522,727(2)$ & 2.0027 & $177,332,733(2)$ & 2.0146 \\
\hline $67(1)$ & -2.0046 & $3(1)$ & -2.0306 & $25(2)$ & 2.0437 & $177,902,862(2)$ & 2.0223 \\
\hline $541(2)$ & 2.0018 & $903(1)$ & -2.0589 & $5,607(1)$ & -2.0277 & $179,979,095(1)$ & -2.0182 \\
\hline $50,799,238(1)$ & -2.0288 & $2(2)$ & 2.0515 & $0,687(2)$ & 2.0363 & $7(2)$ & 2.1018 \\
\hline $55,221,454(2)$ & 2.0242 & $2(2)$ & 2.0616 & 157 & -2.0329 & $5(1)$ & -2.0401 \\
\hline $56,948,780(2)$ & 2.0177 & 121 & 2.1719 & $157,755,123(1)$ & -2.0205 & 182,105, & 2.0084 \\
\hline, $663(1)$ & -2.0081 & 12 & 072 & $158,298,485(2)$ & 2.0273 & $182,223,560(2)$ & 2.0156 \\
\hline $61,331,7$ & 543 & 12 & -2.0 & $160,369,0$ & 2.0071 & $5(2)$ & 2.0195 \\
\hline 69, & .0637 & $127,936,513(1)$ & -2.1105 & $162,962,787(1)$ & -2.0115 & $191,165,600(2)$ & 2.0283 \\
\hline $75,052,114(1)$ & -2.0045 & $128,710,278(2)$ & 2.0444 & $163,724,709(1)$ & -2.0163 & $191,297,535(5)$ & -2.1490 \\
\hline $79,545,241(2)$ & 2.0113 & $129,398,903(2)$ & 2.0431 & $164,198,114(2)$ & 2.0235 & $192,485,616(1)$ & -2.0416 \\
\hline $79,652,248(2)$ & 2.0066 & $130,461,097(2)$ & 2.0963 & $164,689,301(1)$ & -2.1579 & $193,264,636(6)$ & 2.0055 \\
\hline $83,088,043(1)$ & -2.1328 & $131,331,948(2)$ & 2.0047 & $164,880,229(2)$ & 2.0308 & $194,696,968(1)$ & -2.0664 \\
\hline $83,689,523(2)$ & 2.0775 & $137,334,072(2)$ & 2.0239 & $166,201,932(1)$ & -2.0024 & $195,876,805(1)$ & -2.0143 \\
\hline $85,348,958(1)$ & -2.0095 & $137,832,603(1)$ & -2.0134 & $68,573,836(1)$ & -2.0159 & $195,916,549(2)$ & 2. \\
\hline $86,513,820(1)$ & -2.0154 & $138,799,472(2)$ & 2.0135 & $169,750,763(1)$ & -2.1036 & $196,395,161(2)$ & 2.0326 \\
\hline $87,947,597(2)$ & 2.0523 & $139,027,791(1)$ & -2.0031 & $170,375,507(1)$ & -2.0009 & $196,676,303(1)$ & -2.0135 \\
\hline $88,600,095(1)$ & -2.1394 & $141,617,806(1)$ & -2.1253 & $170,704,880(2)$ & 2.0249 & $197,889,883(2)$ & 2.0034 \\
\hline $93,681,183(1)$ & -2.0165 & $144,454,931(1)$ & -2.0380 & $172,000,993(2)$ & 2.0608 & 198,014, & -2.0333 \\
\hline $100,316,552(2)$ & 2.0233 & $145,402,380(2)$ & 2.0012 & $173,289,941(1)$ & -2.0378 & $199,235,289(1)$ & -2.0205 \\
\hline
\end{tabular}

\section{TABLE 2}

Various types of exceptions to Rosser's rule and their frequencies in $\left[g_{-1}, g_{200,000,000}\right)$.

\begin{tabular}{|c|c|c|c|c|c|c|c|c|c|}
\hline \multirow{3}{*}{$\mathrm{g}_{\mathrm{n}}$} & \multirow{3}{*}{$\begin{array}{l}g_{n-1} \\
\psi\end{array}$} & \multicolumn{3}{|c|}{\begin{tabular}{|l} 
Gram block of \\
length 2 without \\
any zeros
\end{tabular}} & & & & type & frequency \\
\hline & & $g_{n}$ & $g_{n+1}$ & & $n+2$ & $g_{n+3}$ & $g_{n+4}$ & & \\
\hline & & $\psi 0$ & $\psi$ & 0 & 3 & $\psi$ & $\Psi$ & 1 & 53 \\
\hline & 3 & 0 & 0 & 0 & & & & 2 & 47 \\
\hline & & 0 & 0 & 0 & 4 & 0 & & 3 & 1 \\
\hline 0 & 4 & 0 & 0 & 0 & & & & 4 & 0 \\
\hline & & 0 & & 0 & 2 & 2 & & 5 & 1 \\
\hline 2 & 2 & 0 & & 0 & & & & 6 & 2 \\
\hline
\end{tabular}


Very recently, Karkoschka and Werner [8] have developed a method for detecting exceptions to Rosser's rule with relatively small computational effort, i.e., by searching in certain selected small ranges of a given $t$-interval. A comparison of their results with Table 1 shows the power of their method: in $\left[g_{3,500,000}, g_{50,000,000}\right)$ they found all 9 exceptions to Rosser's rule, and in $\left[g_{100,000,000}, g_{120,000,000}\right)$ they found 6 of the 9 exceptions.

Table 3 is a continuation of Table 1 of Brent [2]. Six Gram blocks of length 8 were found. The average block length up to $n=200,000,000$ is 1.1951 . We have compared the results of L R \& W with those of Brent in the range $\left[g_{110,000,000}, g_{120,000,002}\right)$. The observed differences were extremely small. Brent's program counted 7,011,482 Gram blocks of length $1,1,055,511$ of length 2 and 230,234 of length 3 . The corresponding figures obtained by L R \& W were 7,011,494, 1,055,508 and 230,232, respectively. The numbers of Gram blocks of length $\geqslant 4$ were the same for both programs.

TABLE 3

(continuation of Table 1 of Brent [2])

Number of Gram blocks of given length

\begin{tabular}{ccccccccc}
\hline $\mathrm{n}$ & $\mathrm{J}(1, \mathrm{n})$ & $\mathrm{J}(2, \mathrm{n})$ & $J(3, n)$ & $J(4, n)$ & $J(5, n)$ & $J(6, n)$ & $J(7, n)$ & $J(8, n)$ \\
\hline $80,000,000$ & $56,942,025$ & $8,886,072$ & $1,714,271$ & 260,637 & 18,807 & 1,033 & 34 & \\
$90,000,000$ & $63,977,026$ & $9,439,917$ & $1,941,455$ & 299,932 & 22,257 & 1,240 & 46 & \\
$100,000,000$ & $71,004,697$ & $10,493,487$ & $2,169,610$ & 340,360 & 25,813 & 1,436 & 54 & \\
$110,000,000$ & $78,023,506$ & $11,547,936$ & $2,399,154$ & 381,216 & 29,601 & 1,644 & 61 & \\
$120,000,000$ & $85,034,988$ & $12,603,447$ & $2,629,388$ & 422,721 & 33,500 & 1,841 & 74 & 1 \\
$130,000,000$ & $92,041,326$ & $13,659,023$ & $2,860,087$ & 464,955 & 37,495 & 2,070 & 92 & 1 \\
$140,000,000$ & $99,041,526$ & $14,713,754$ & $3,092,451$ & 507,686 & 41,631 & 2,332 & 102 & 4 \\
$150,000,000$ & $106,038,874$ & $15,768,532$ & $3,325,400$ & 550,630 & 45,795 & 2,591 & 114 & 3 \\
$156,800,000$ & $110,793,769$ & $16,486,479$ & $3,484,026$ & 579,999 & 48,731 & 2,780 & 120 & 3 \\
$200,000,000$ & $140,956,084$ & $21,047,520$ & $4,497,856$ & 771,607 & 68,631 & 4,031 & 213 & 6 \\
\hline
\end{tabular}

TABLE 4

(continuation of Table 2 of Brent [2])

Number of Gram intervals containing exactly $m$ zeros

\begin{tabular}{cccccc}
\hline $\mathrm{n}$ & $\mathrm{m}=0$ & $\mathrm{~m}=1$ & $\mathrm{~m}=2$ & $\mathrm{~m}=3$ & $\mathrm{~m}=4$ \\
\hline $80,000,000$ & $10,513,316$ & $59,105,832$ & $10,248,390$ & 132,461 & 1 \\
$90,000,000$ & $11,854,362$ & $66,440,792$ & $11,555,331$ & 149,514 & 1 \\
$100,000,000$ & $13,197,331$ & $73,771,910$ & $12,864,188$ & 166,570 & 1 \\
$110,000,000$ & $14,543,760$ & $81,096,629$ & $14,175,463$ & 184,147 & 1 \\
$120,000,000$ & $15,892,224$ & $88,416,806$ & $15,489,718$ & 201,251 & 1 \\
$130,000,000$ & $17,242,449$ & $95,733,829$ & $16,804,996$ & 218,725 & 1 \\
$140,000,000$ & $18,594,089$ & $103,047,955$ & $18,121,824$ & 236,131 & 1 \\
$150,000,000$ & $19,946,624$ & $110,360,313$ & $19,439,504$ & 253,558 & 1 \\
$156,800,000$ & $20,867,682$ & $115,330,181$ & $20,336,593$ & 265,543 & 1 \\
$200,000,000$ & $26,731,720$ & $146,878,417$ & $26,048,007$ & 341,855 & 1 \\
\hline
\end{tabular}


Table 4 is a continuation of Table 2 of Brent [2]. The percentages of the numbers of Gram intervals up to $n=200,000,000$ containing exactly $m$ zeros are 13.4, 73.4, 13.0, and 0.2 for $m=0,1,2$, and 3, respectively. No new Gram intervals with exactly four zeros were found.

Table 5 continues Table 4 of Brent [2]. As yet, no Gram block of type $(7,1)$ was found. Due to the shifts, we may have missed earlier occurrences of blocks of types $(7,7),(8,3)$ and $(8,7)$, although we consider this unlikely.

TABLE 5

(continuation of Table 4 of Brent [2])

First occurrences of Gram blocks of various types

\begin{tabular}{ccl}
\hline $\mathrm{j}$ & $\mathrm{k}$ & \multicolumn{1}{c}{$\mathrm{n}$} \\
\hline 7 & 7 & $195,610,937$ (L R \& W) \\
8 & 2 & $112,154,948$ (BRENT) \\
8 & 3 & $175,330,804$ (L R \& W) \\
8 & 6 & $145,659,810$ (BRENT) \\
8 & 7 & $165,152,519$ (L R \& W) \\
\hline
\end{tabular}

TABLE 6

Number of Gram blocks of type $(j, k), 1 \leqslant j \leqslant 8,1 \leqslant k \leqslant j$, in the interval $\left[g_{156,800,000}, g_{200,000,000}\right)$

\begin{tabular}{|c|c|c|c|c|c|c|c|c|c|}
\hline$\downarrow j$ & $\begin{array}{c}\mathrm{k} \\
1\end{array}$ & 2 & 3 & 4 & 5 & 6 & 7 & 8 & total \\
\hline 1 & $30,162,315$ & & & & & & & & $30,162,315$ \\
\hline 2 & $\begin{array}{r}2,279,942 \\
(50)\end{array}$ & $\begin{array}{r}2,281,053 \\
(50)\end{array}$ & $\begin{array}{r}43 \text { blocks } \\
3 \text { blocks }\end{array}$ & $\begin{array}{l}\text { with } \\
\text { with } 2\end{array}$ & $\begin{array}{ll}0 & 0 \\
2 & 2\end{array}$ & $\begin{array}{l}\text { zero-pat } \\
\text { zero-pat }\end{array}$ & $\begin{array}{l}\text { ttern } \\
\text { ttern }\end{array}$ & & $4,561,041$ \\
\hline 3 & $\begin{array}{c}479,720 \\
(47)\end{array}$ & $\begin{array}{r}53,497 \\
(5)\end{array}$ & $\begin{array}{c}480,613 \\
(47)\end{array}$ & & & & & & $1,013,830$ \\
\hline 4 & $\begin{array}{r}87,367 \\
(46)\end{array}$ & $\begin{array}{r}8,592 \\
(4)\end{array}$ & $\begin{array}{r}8,499 \quad 8 \\
(4)\end{array}$ & $\begin{array}{r}37,150 \\
(45)\end{array}$ & & & & & 191,608 \\
\hline 5 & $\begin{array}{r}7,581 \\
(38)\end{array}$ & $\begin{array}{r}1,811 \\
(9)\end{array}$ & $\begin{array}{l}948 \\
(5)\end{array}$ & $\begin{array}{r}1,882 \\
(9)\end{array}$ & $\begin{array}{c}7,678 \\
(39)\end{array}$ & & & & 19,900 \\
\hline 6 & $\begin{array}{l}156 \\
(12)\end{array}$ & $\begin{array}{r}337 \\
(27)\end{array}$ & $\begin{array}{l}119 \\
(10)\end{array}$ & $\begin{array}{l}126 \\
(10)\end{array}$ & $\begin{array}{r}366 \\
(29)\end{array}$ & $\begin{array}{r}147 \\
(12)\end{array}$ & & & 1,251 \\
\hline 7 & 0 & 29 & 17 & 3 & 17 & 26 & 1 & & 93 \\
\hline 8 & 0 & 0 & $\left.1^{\star}\right)$ & 0 & 0 & $1^{\star)}$ & $\left.1^{\star}\right)$ & 0 & 3 \\
\hline
\end{tabular}

$\left.{ }^{\star}\right)_{\text {viz. }} \mathrm{B}_{\mathrm{n}}$, for $\mathrm{n}=175,330,804,181,390,731$ and $165,152,519$.

In Table 6 we list the number of Gram blocks of type $(j, k), 1 \leqslant j \leqslant 8,1 \leqslant k \leqslant j$, in the interval $\left[g_{156,800,000}, g_{200,000,000}\right)$, as they were actually counted by the L R \& $\mathrm{W}$-program. On the line with $j=2$ we also mention the numbers of Gram blocks of length 2 with zero-pattern " 00 " and those with pattern " 2 " which could, of course, neither be classified as type $(2,1)$ nor as $(2,2)$. The 43 blocks with " 00 "pattern correspond to the exceptions to Rosser's rule in $\left[g_{156,800,000}, g_{200,000,000}\right)$ and 
the 3 blocks with " 2 2"-pattern correspond to the exceptions of types 5 and 6 (cf. Table 2). The entries in parentheses give the approximate percentages with respect to the total number of blocks of length $j$, given in the final column.

Our main purpose in presenting this table is to render support to the $L \mathbf{R} \&$ W-strategy of dealing with Gram blocks of length $j \geqslant 2$. The table shows that this strategy is successful for $2 \leqslant j \leqslant 5$. However, for $j \geqslant 6$ the missing two zeros show an increasing tendency to lie either in $\left(g_{n+1}, g_{n+2}\right)$ or in $\left(g_{n+j-2}, g_{n+j-1}\right)$. Only one of the 93 blocks of length $j=7$ has its missing two zeros in one of the outer Gram intervals!

Department of Computer Science

The Australian National University

P. O. Box 4

Canberra ACT 2600, Australia

Mathematical Centre

Kruislaan 413

1098 SJ Amsterdam, The Netherlands

1. R. Backlund, "Sur les zéros de la fonction $\zeta(s)$ de Riemann," C. R. Acad. Sci. Paris, v. 158, 1914, pp. 1979-1982.

2. R. P. BRent, "On the zeros of the Riemann zeta function in the critical strip," Math. Comp., v. 33, 1979, pp. 1361-1372.

3. R. P. BRent \& L. SChoenfeld, Numerical Approximation of the Riemann Zeta Function, Technical Report, Dept. of Computer Science, The Australian National University. (To appear.)

4. H. M. Edwards, Riemann's Zeta Function, Academic Press, New York, 1974.

5. W. GABCKE, Neue Herleitung und explizite Restabschätzung der Riemann-Siegel-Formel, Dissertation, Universität Göttingen, 1979.

6. J. Gram, "Sur les zéros de la fonction $\zeta(s)$ de Riemann," Acta Math., v. 27, 1903, pp. 289-304.

7. J. I. Hurchinson, "On the roots of the Riemann zeta-function," Trans. Amer. Math. Soc., v. 27, 1925, pp. 49-60.

8. E. KarkoschKa \& P. Werner, "Einige Ausnahmen zur Rosserschen Regel in der Theorie der Riemannschen Zetafunktion," Computing, v. 27, 1981, pp. 57-69.

9. R. S. Lehman, "Separation of zeros of the Riemann zeta-function," Math. Comp., v. 20, 1966, pp. 523-541.

10. D. H. Lehmer, "On the roots of the Riemann zeta function," Acta Math., v. 95, 1956, pp. 291-298.

11. D. H. Lehmer, "Extended computation of the Riemann zeta function," Mathematika, v. 3, 1956, pp. $102-108$.

12. J. van de Lune, H. J. J. Te Riele \& D. T. Winter, Rigorous High Speed Separation of Zeros of Riemann's Zeta Function, Report NW 113/81, Mathematical Centre, Amsterdam, October 1981.

13. N. A. Meller, "Computations connected with the check of Riemann's hypothesis," Dokl. Akad. Nauk SSSR, v. 123, 1958, pp. 246-248. (Russian)

14. J. B. Rosser, J. M. YOHE \& L. SCHOENFELD, "Rigorous computation and the zeros of the Riemann zeta-function," Proc. IFIP Congress, Edinburgh, 1968.

15. E. C. Titchmarsh, "The zeros of the Riemann zeta function," Proc. Roy. Soc. London, v. 151, 1935, pp. 234-255; v. 157, 1936, pp. 261-263.

16. E. C. TItchmarsh, The Theory of the Riemann Zeta-Function, Clarendon Press, Oxford, 1951. 